\title{
Influences of vascular niches on hematopoietic stem cell fate
}

\author{
Yuya Kunisaki · Paul S. Frenette
}

Received: 26 March 2014/ Accepted: 31 March 2014/Published online: 23 April 2014

(c) The Japanese Society of Hematology 2014

\begin{abstract}
The fate decision of hematopoietic stem cells (HSCs), quiescence, proliferation or differentiation, is uniquely determined by functionally specialized microenvironments defined as the HSC niches. However, whether quiescence and proliferation of HSCs are regulated by spatially distinct niches is unclear. Although various candidate stromal cells have been identified as potential niche cells, the spatial localization of quiescent HSCs in the bone marrow remains controversial. In our recent study, we have established whole-mount confocal immunofluorescence techniques, which allow us to precisely assess the localization of HSCs and their relationships with stromal structures. Furthermore, we have assessed the significance of these associations using a computational simulation. These novel analyses have revealed that quiescent HSCs are specifically associated with small caliber arterioles, which are predominantly distributed in the endosteal bone marrow while the associations with sinusoidal vessels or osteoblasts are not significant. Physical ablation of the
\end{abstract}

Y. Kunisaki · P. S. Frenette $(\square)$

Ruth L. and David S. Gottesman Institute for Stem Cell and

Regenerative Medicine Research, Bronx, NY 10461, USA

e-mail: paul.frenette@einstein.yu.edu

Y. Kunisaki · P. S. Frenette

Department of Cell Biology, Albert Einstein College of

Medicine, Bronx, NY 10461, USA

Y. Kunisaki · P. S. Frenette

Department of Medicine, Albert Einstein College of Medicine,

Bronx, NY 10461, USA

Present Address:

Y. Kunisaki $(\square)$

Department of Medicine and Biosystemic Science, Kyushu

University, Fukuoka, Fukuoka 812-8582, Japan

e-mail: kunisaki@cancer.med.kyushu-u.ac.jp arteriolar niche causes the shift of HSC localization to sinusoidal niches, where HSCs are switched into non-quiescent status. This new imaging analyses together with previous studies suggest the presence of spatially distinct vascular niches for quiescent and non-quiescent (proliferating) HSCs in the bone marrow.

Keywords Hematopoietic stem cells · Quiescence · Arterioles

\section{Introduction}

Hematopoietic stem cells (HSCs) maintain hematopoietic homeostasis through lifelong replenishment of all blood cell lineages by a finely tuned orchestration of self-renewal and differentiation controlled by specific cellular and molecular microenvironments defined as niches [1]. The "niche" concept has been validated by numerous studies since Schofield postulated its presence in 1978 [2]. Most of HSCs divide infrequently and are quiescent in the niche [3]. However, they are reversibly activated in response to hematopoietic stresses [4]. Cell cycle quiescence is a key behavior of stem cells, which protects them from being exhausted by exogenous insults [5] and is also assumed to prevent them from acquiring genetic mutations that potentially result in consequent malignant transformations [6]. Although quiescence and proliferation of HSCs are thought to be regulated by their microenvironments, it remains unclear whether and if so how spatially distinct niches control cell cycle status.

The identification of cellular constituents of the HSC niche has recently been the subject of intense investigations. Osteoblasts have been proposed to promote HSC quiescence via direct contact [7] and the secretion of 
angiopoietin-1 [8] or osteopontin [9, 10]. On the other hand, an emerging role of the bone marrow vasculature has recently gained support and interest [11] as other studies have found that most HSCs are localized adjacent to blood vessels (sinusoids), near perivascular cell populations characterized as CXCL12-abundant reticular (CAR) cells $[12,13]$, Nestin $^{+}$mesenchymal stem cells [14], and Leptin receptor $\left(\right.$ LEPR) ${ }^{+}$cells [15]. Based on the previous studies, a prevalent unifying interpretation of the literature has been that the osteoblastic and vascular niches could confer distinct microenvironments promoting HSC quiescence and proliferation, respectively. However, this popular concept has not been supported by rigorous analyses. Recent studies further have reported that endothelial cells and perivascular stromal cells are sources of the factors such as CXCL12 and stem cell factor (SCF), known to be essential for HSC retention and maintenance [14-16]. Series of studies also have shown that genetic deletion of the factor, CXCL12, in osteoblasts [17] and ablation of osteoblasts (David T. Scadden personal communication) affect lymphoid-committed progenitors rather than HSCs. On the other hand, the proximity of HSCs to sinusoidal vessels, which has been reported in many previous studies, might be biased by the abundance and dense network of sinusoids in the bone marrow. The relationship between HSCs and arteries has been well documented in the emergence of HSCs during development as definitive hematopoiesis is beginning in the aorta-gonad-mesonephros (AGM) region [18]. However, it has been unknown whether arteries play specific roles for HSCs in adult bone marrow.

To address these issues, we have established a wholemount immunofluorescence imaging technique in which the three-dimensional (3D) spatial relationships between stromal structures and HSCs in the bone marrow can be precisely determined [19]. In this article, we will introduce the advance of HSC niche imaging and propose a new model of "the HSC niches" that can be drawn from recent studies.

\section{New imaging analyses of hematopoietic stem cell niches}

The visualization of HSCs is the most direct way to find their niches in the bone marrow. For the last decades, great advances in bone marrow imaging techniques have been achieved. To analyze the spatial localization of HSCs in the bone marrow, prior studies used exogenously labeled HSCs and transplanted them into mice to visualize them in the skull bone marrow in live animals by intravital microscopy [20-22]. The live sequential imaging technique allows one to assess the 3D actual cell movement (=cell migration). However, this approach is limited by the extensive manipulation of HSCs and the narrow discrimination that intravital procedures in the skull bone marrow provide to discern vascular and osteoblastic niches. An alternative approach has been to stain endogenous HSCs in sectioned bone marrow using a combination of surface markers, CD150, CD48 and CD41, through which HSCs can be highly enriched [12, 13, 23, 24], but thin sections provide little 3D information about the relationship with various bone marrow structures. To overcome the issue for the bone marrow 3D structure, a whole-mount confocal immunofluorescence technique in mouse sternal and longbone bone marrow has been developed [23, 25]. To gain more insight in the 3D structure of the HSC niche, we have adopted and improved the imaging technique by which the 3D spatial relationships between vascular and stromal structures and endogenous $\mathrm{CD} 150^{+} \mathrm{CD} 48^{-} \mathrm{CD} 41^{-}$Lineage $^{-}$HSCs in the sternal or femoral bone marrow can be analyzed [19] (Fig. 1).

\section{Computational modeling of HSC localization in the bone marrow}

This new imaging technique further allows us to perform a computational simulation on the images of whole-mount prepared sterna to assess the significance of the association between HSCs and bone marrow structures we have observed in situ. In a null model, HSCs are randomly placed on the unoccupied regions of binary spatial maps of bone marrow structures, sinusoids, arterioles and bone lining osteoblasts, defined from the images of whole-mount prepared sterna, and Euclidean distance of the random HSCs to the structures was measured. The means of 1,000 simulations defined a distribution of mean distances one would observe for non-preferentially localized HSCs in relation to the respective structures. If the in situ distance measurements were not statistically different from those obtained by a random placement of HSCs on the same structures in silica, this would indicate a non-preferential spatial HSC distribution (Fig. 2).

This novel method using the combination of the wholemount imaging technique and computational modeling allows for precise measurements of 3D distances of HSCs from structures and computational simulations to define the significance of these interactions.

\section{Arterioles and sinusoids are ensheathed by distinct perivascular cells}

Whole-mount confocal immunofluorescence assessment of vascular structure revealed an evenly distributed sinusoidal network and rare small caliber arterioles, which were found predominantly in close proximity to the bone in the bone 


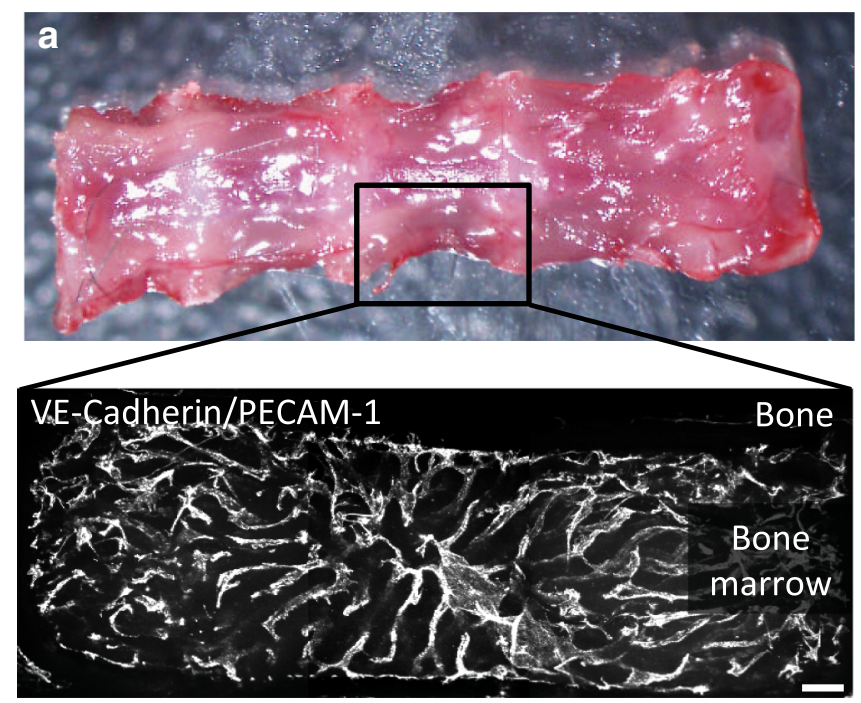

Fig. 1 A whole-mount immunofluorescence imaging of HSC niches. a Whole-mount immunofluorescence techniques of the sternal bone marrow. A picture of mouse sternum and representative images of the vasculature of the sternal bone marrow stained with anti-VE-cadherin

marrow (Fig. 2a) [19]. Recent studies have highlighted the critical importance of bone marrow stromal cells as essential constituents for the HSC niche through production of factors such as CXCL12, SCF. Nestin ${ }^{+}$and leptin receptor $(\mathrm{LEPR})^{+}$perivascular stromal cells have been identified as HSC niche cells $[14,15]$. Nestin ${ }^{+}$perivascular cells contain all bone marrow mesenchymal stem cell activity [26]. Our previous study has identified distinct subsets of $\mathrm{Nestin}^{+}$cells that are associated with distinct vessel types: rare Nestin-GFP ${ }^{\text {bright }}$ cells $(\sim 10 \%$ of Nestin $^{+}$cells) exhibit a pericyte morphology, express the NG2 antigen but not LEPR, and are exclusively associated with arterioles, whereas Nestin-GFP ${ }^{\mathrm{dim}}$ cells ( $\sim 80 \%$ of Nestin $^{+}$cells) exhibit a reticular morphology, are $\mathrm{NG}^{-}$and $\mathrm{LEPR}^{+}$, and are associated with sinusoids [19].

\section{Mesenchymal and osteo-precursor cells as niche components}

Nestin-GFP $^{+}$cells overlap with $\mathrm{LEPR}^{+}$perivascular cells and both of the populations contain mesenchymal progenitor (CFU-F) activity [27]. While LEPR $^{+}$cells have been shown to represent an important source of SCF that is essential for HSC maintenance [15], deletion of CXCL12 in $\mathrm{LEPR}^{+}$cells has little effect on HSC maintenance but leads to HSC mobilization to the spleen [17]. Interestingly, a similar phenotype was observed with the deletion of CXCL12 using Osterix-cre [16]. The Osterix (Osx) transcription factor regulates osteoblast differentiation as revealed by defective bone formation in Osx-deficient mice

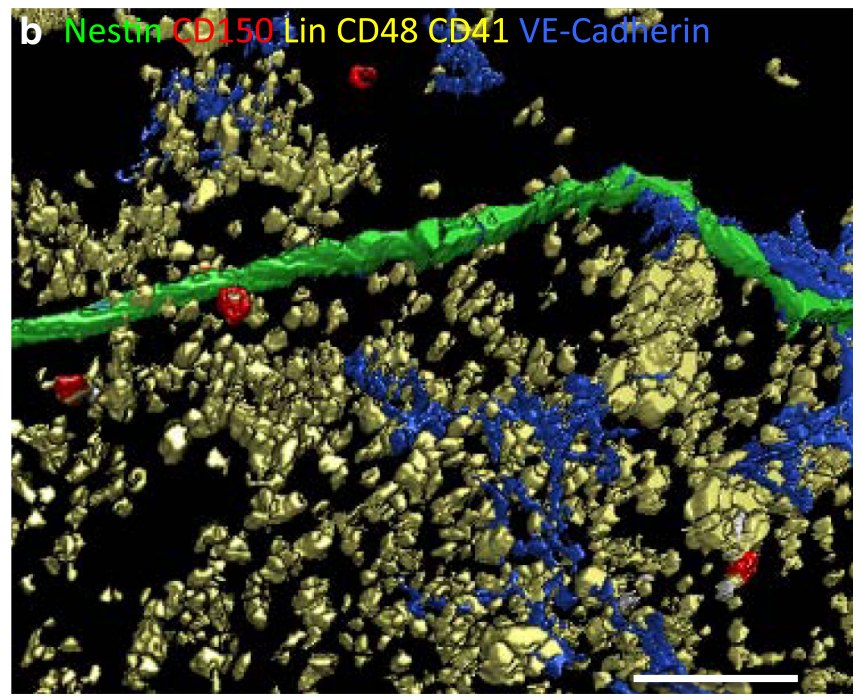

and PECAM1 antibodies. Scale bar $100 \mu \mathrm{m}$. b 3D-reconstructed images of the sternal bone marrow. Phenotypic HSCs are identified as $\mathrm{CD} 150^{+} \mathrm{CD}^{-} 1^{-} \mathrm{CD} 48^{-}$Lineage $^{-}$cells. Scale bar $50 \mu \mathrm{m}$

[28]. Osteoblasts, descendants of MSCs, have also been suggested as niche cells for HSCs and progenitors [8, 29, 30]. Gain-of-function and loss-of-function approaches showed that an increase in the number of osteoblasts correlated with increased numbers of HSCs [29-31]. Our recent lineage tracing analyses using tamoxifen-inducible Osx-cre (OsX-cre ${ }^{\text {ERT2 }}$ ) have revealed that expression of Osx is not restricted in osteoblasts and that Osx-expressing cells at the neonatal stage are bone marrow stromal precursors [32]. Owen and Friedenstein [33] predicted 25 years ago that the differentiation tree of stromal cells of the bone marrow is as complex as their hematopoietic counterparts. However, the hierarchical organization of stromal cells remains unknown. In addition, stromal cells marked by various genetic reporter mouse strains, e.g., Prx1-cre, Osterix-cre, NG2-cre ${ }^{\mathrm{ERTM}}$, have been reported as important niche constituents [13, 16, 17, 19]. Since they seem to have redundancy to some extent [34], future studies using combination of these genetic models will lead us to understand the hierarchy of bone marrow stromal cells contributing fate decision of HSCs and progenitors.

\section{$\mathrm{NG2}^{+}$arteriolar pericytes form niches for dormant hematopoietic stem cells}

In our recent study using these imaging techniques [19], we found that phenotypic $\mathrm{CD} 150^{+} \mathrm{CD} 48^{-} \mathrm{CD} 41^{-}$Lineage $^{-}$ HSCs are specifically associated with arterioles, which are predominantly distributed in the endosteal region in the bone marrow, while the spatial associations of HSCs with 
a

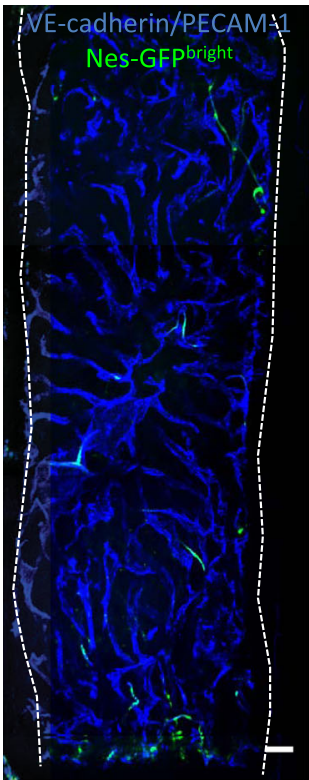

Arteriole

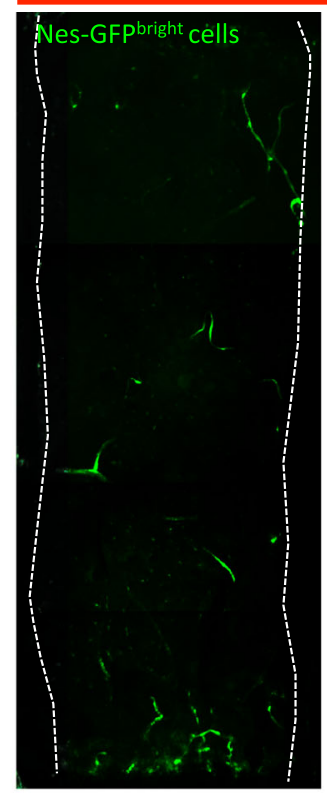

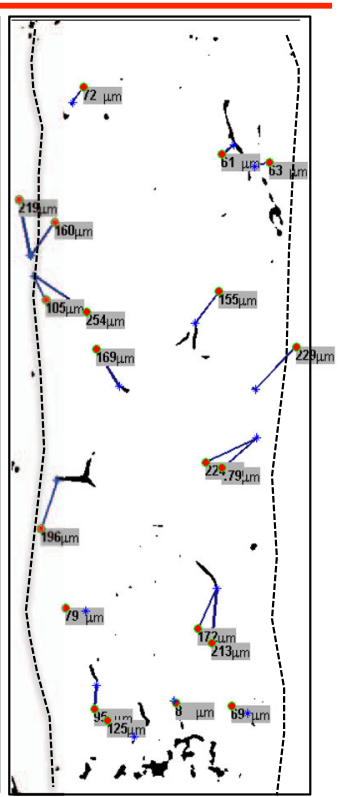

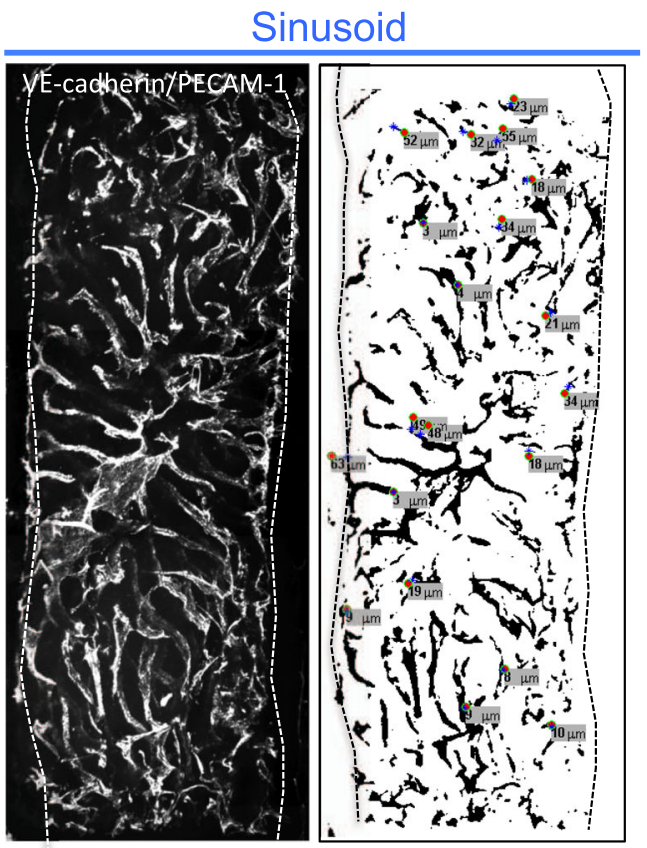

b

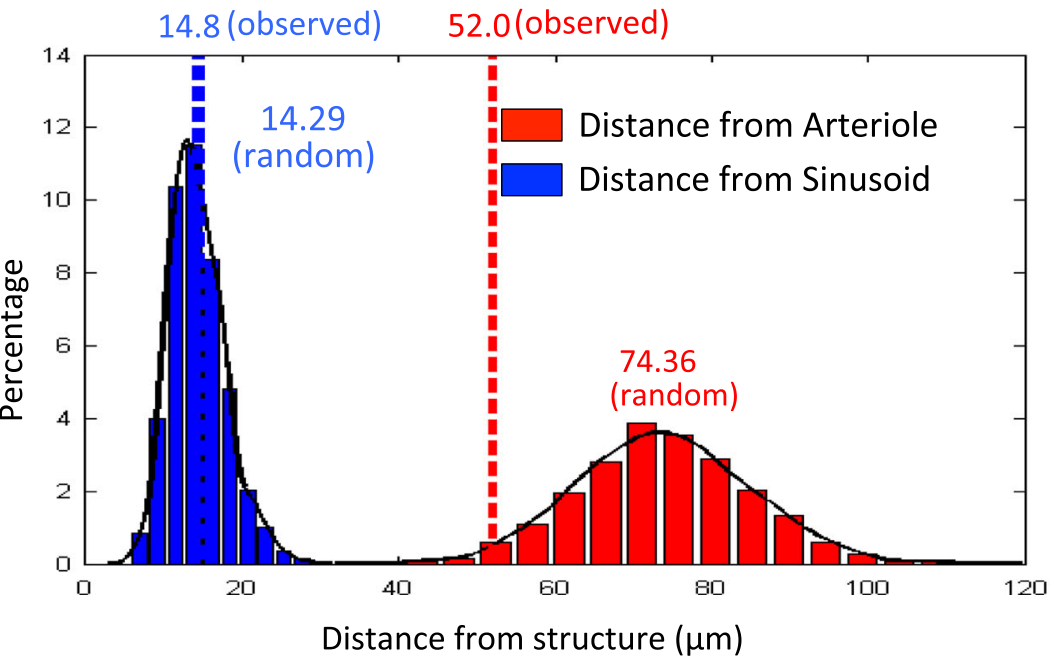

Fig. 2 A computational modeling of the HSC localization in relation to bone marrow arterioles or sinusoids. a Computational simulation of randomly distributed HSCs on images of whole-mount prepared sterna. To establish the null model, binary spatial maps of the sinusoids, arterioles were defined from the images of whole-mount prepared sterna. To simulate a null model in which HSCs are not preferentially localized in the marrow, we randomly placed $20 \mathrm{HSCs}$ (to reflect the mean HSCs/sternum observed in situ) on the unoccupied regions of the spatial maps and measured the Euclidean distance of HSCs to the nearest vascular structure. Scale bar $100 \mu \mathrm{m}$.

sinusoids or osteoblasts, which have been reported in previous studies, are statistically indistinguishable from random distribution. Furthermore, we analyzed the localization of quiescent (dormant) and non-quiescent HSCs in relation to arterioles, sinusoids or osteoblasts. Quiescence of HSCs is evaluated using the nuclear proliferation marker, Ki-67 staining. Prolonged quiescence is b The means of 1,000 simulations defined a distribution of mean distances one would observe for non-preferentially (random) localized HSCs in relation to the respective structures. If the in situ distance measurements are not statistically significantly different from those obtained by a random placement of HSCs on the same structures in silica, this would indicate a non-preferential spatial HSC distribution. The cumulative probability of observing the in situ mean was calculated based on the normal distribution obtained from our simulation on a map of each bone marrow structure

commonly referred as dormancy, which can be tracked as label-retaining cells. 3D whole-mount immunofluorescence imaging analyses combined with these quiescence (dormant) markers showed that the association of quiescent, but not non-quiescent, HSCs with arterioles was significant whereas there was no significant difference in the distribution of quiescent and non-quiescent HSCs in relation 


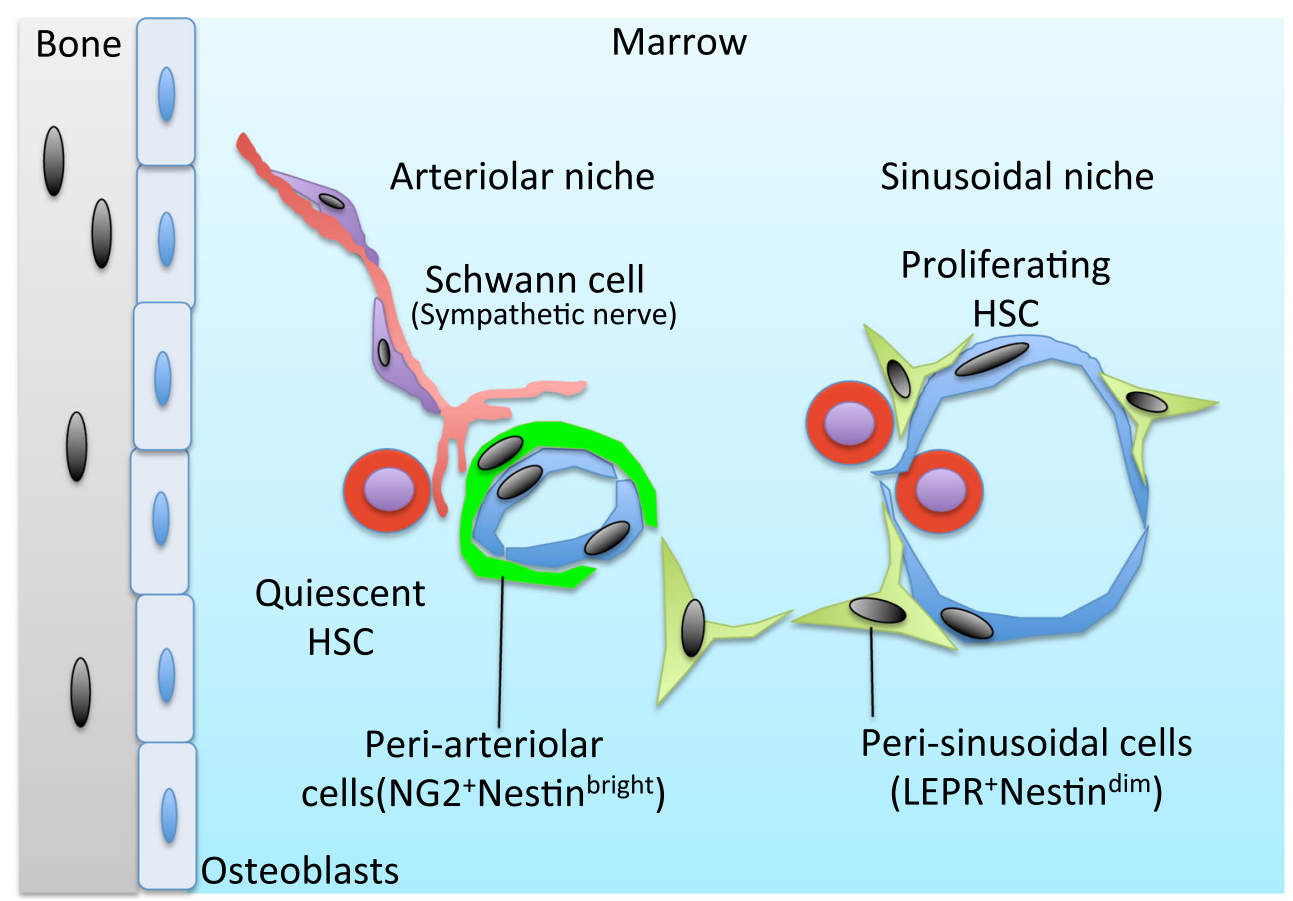

Fig. 3 A scheme of the HSC vascular niches in the bone marrow. Bone marrow vasculature is composed of a dense evenly distributed sinusoidal network and rare small caliber arterioles, which are predominantly distributed in the endosteal region. Arterioles and sinusoids are ensheathed by distinct perivascular $\mathrm{NG}^{+}$Nestin-

with osteoblasts. Notably, we found that peri-arteriolar Nes-GFP ${ }^{\text {bright }}$ cells themselves were more quiescent than peri-sinusoidal Nes-GFP ${ }^{\mathrm{dim}}$ cells. After 5FU administration, the number of Nes-GFP ${ }^{\text {bright }}$ cells was largely preserved compared to Nes-GFP ${ }^{\mathrm{dim}}$ cells and the vast majority of HSCs were closely associated with arterioles. These results indicate that bone marrow arterioles comprise a specialized microenvironment that promotes quiescence of both arteriolar niche cells and the HSCs. It is also suggested that there is an efficient communication between HSC and the niche as pharmacological or genetic alteration of HSC cycle status (e.g., poly IC administration [4] or Pml-deficient mice [35]) can lead to changes in HSC distribution in the bone marrow [19]. It is possible that altered expression of receptors on HSCs (e.g., c-kit or $\alpha 4$-integrin as previously reported [36-38]), upon cell cycle activation may also contribute to the translocation from a quiescent to a proliferative niche. In addition to this spatial relationship, physical depletion of Nestin-GFP ${ }^{\text {bright }} \mathrm{NG}^{+}$cells changed HSC localization away from arterioles, and switched them into a non-quiescent state with following reduction in the number and frequency of HSCs in the bone marrow. Taken together, these results indicate that peri-arteriolar $\mathrm{NG}^{+}$ cells form niches for dormant HSCs.
$\mathrm{GFP}^{\text {bright }}$ or LEPR ${ }^{+}$Nestin-GFP ${ }^{\mathrm{dim}}$, cells, respectively. Bone marrow arterioles and the pericytes comprise a specialized microenvironment, the arteriolar niche that promotes quiescence of HSCs whereas sinusoids form another niche, which also play major roles in HSC proliferation and trafficking, such as recruitment or mobilization

\section{A model of the hematopoietic stem cell niches drawn by recent studies}

These results thus suggest that distinct vascular niches, quiescent or proliferative, are conferred by distinct blood vessel types (Fig. 3). The presence of distinct vascular HSC niches also implies important functional differences of these niches with regards to HSC migration. It is unlikely, for example, that HSCs would traffic through arterioles, owing to their thickness, high pressure, and low density of adhesion molecule expression. By contrast, sinusoids are suited for hematopoietic cell migration due to their fenestration and high expression of adhesion molecules, including selectins and VCAM1 that mediate homing [39-42]. It is thus conceivable that a continuous exchange between the sinusoidal (mobilizable and proliferative) and arterial (quiescent and dormant) niches contributes to maintain a tightly controlled balance between proliferation and dormancy in HSCs. A recent study demonstrates that E-selectin, an adhesion molecule constitutively expressed in certain bone marrow sinusoidal endothelial cells [20], promotes HSC proliferation [43], which supports the idea that sinusoids may represent a proliferative niche. 
This vascular niche model, in which arterioles and sinusoids differentially regulate HSC quiescence and proliferation, respectively, will have implications on the behavior of healthy HSC and may be useful in the future for evaluating the niches for cancer (leukemic) stem cells.

Conflict of interest The authors declare no competing financial interests.

\section{References}

1. Kunisaki Y, Frenette PS. The secrets of the bone marrow niche: enigmatic niche brings challenge for HSC expansion. Nat Med. 2012;18:864-5.

2. Schofield R. The relationship between the spleen colony-forming cell and the haemopoietic stem cell. Blood Cells. 1978;4:7-25.

3. Kiel MJ, He S, Ashkenazi R, et al. Haematopoietic stem cells do not asymmetrically segregate chromosomes or retain BrdU. Nature. 2007;449:238-42.

4. Wilson A, Laurenti E, Oser G, et al. Hematopoietic stem cells reversibly switch from dormancy to self-renewal during homeostasis and repair. Cell. 2008;135:1118-29.

5. Orford KW, Scadden DT. Deconstructing stem cell self-renewal: genetic insights into cell-cycle regulation. Nat Rev Genet. 2008;9:115-28.

6. Lobo NA, Shimono Y, Qian D, Clarke MF. The biology of cancer stem cells. Annu Rev Cell Dev Biol. 2007;23:675-99.

7. Sugimura R, He XC, Venkatraman A, et al. Noncanonical Wnt signaling maintains hematopoietic stem cells in the niche. Cell. 2012;150:351-65.

8. Arai F, Hirao A, Ohmura M, et al. Tie2/angiopoietin-1 signaling regulates hematopoietic stem cell quiescence in the bone marrow niche. Cell. 2004;118:149-61.

9. Nilsson SK, Johnston HM, Whitty GA, et al. Osteopontin, a key component of the hematopoietic stem cell niche and regulator of primitive hematopoietic progenitor cells. Blood. 2005;106: 1232-9.

10. Stier S, Ko Y, Forkert R, et al. Osteopontin is a hematopoietic stem cell niche component that negatively regulates stem cell pool size. J Exp Med. 2005;201:1781-91.

11. Takakura N. Guest editorial: mutual relationship between vascular biology and hematology. Int J Hematol. 2012;95:117-8.

12. Sugiyama $T$, Kohara $H$, Noda M, Nagasawa $T$. Maintenance of the hematopoietic stem cell pool by CXCL12-CXCR4 chemokine signaling in bone marrow stromal cell niches. Immunity. 2006;25:977-88

13. Omatsu Y, Sugiyama T, Kohara H, et al. The essential functions of adipo-osteogenic progenitors as the hematopoietic stem and progenitor cell niche. Immunity. 2010;33:387-99.

14. Mendez-Ferrer S, Michurina TV, Ferraro F, et al. Mesenchymal and haematopoietic stem cells form a unique bone marrow niche. Nature. 2010;466:829-34.

15. Ding L, Saunders TL, Enikolopov G, Morrison SJ. Endothelial and perivascular cells maintain haematopoietic stem cells. Nature. 2012;481:457-62.

16. Greenbaum A, Hsu YM, Day RB, et al. CXCL12 in early mesenchymal progenitors is required for haematopoietic stem-cell maintenance. Nature. 2013;495:227-30.

17. Ding L, Morrison SJ. Haematopoietic stem cells and early lymphoid progenitors occupy distinct bone marrow niches. Nature. 2013;495:231-5

18. Mikkola HK, Orkin SH. The journey of developing hematopoietic stem cells. Development. 2006;133:3733-44.
19. Kunisaki Y, Bruns I, Scheiermann C, et al. Arteriolar niches maintain haematopoietic stem cell quiescence. Nature. 2013;502:637-43.

20. Sipkins DA, Wei $X, W u$ JW, et al. In vivo imaging of specialized bone marrow endothelial microdomains for tumour engraftment. Nature. 2005;435:969-73.

21. Lo Celso C, Fleming HE, Wu JW, et al. Live-animal tracking of individual haematopoietic stem/progenitor cells in their niche. Nature. 2009;457:92-6.

22. Fujisaki J, Wu J, Carlson AL, et al. In vivo imaging of Treg cells providing immune privilege to the haematopoietic stem-cell niche. Nature. 2011;474:216-9.

23. Nombela-Arrieta C, Pivarnik G, Winkel B, et al. Quantitative imaging of haematopoietic stem and progenitor cell localization and hypoxic status in the bone marrow microenvironment. Nat Cell Biol. 2013; 15:533-43.

24. Kiel MJ, Yilmaz OH, Iwashita T, et al. SLAM family receptors distinguish hematopoietic stem and progenitor cells and reveal endothelial niches for stem cells. Cell. 2005;121:1109-21.

25. Takaku T, Malide D, Chen J, et al. Hematopoiesis in 3 dimensions: human and murine bone marrow architecture visualized by confocal microscopy. Blood. 2010;116:e41-55.

26. Mendez-Ferrer S, Lucas D, Battista M, Frenette PS. Haematopoietic stem cell release is regulated by circadian oscillations. Nature. 2008;452:442-7.

27. Pinho S, Lacombe J, Hanoun M, et al. PDGFRalpha and CD51 mark human Nestin ${ }^{+}$sphere-forming mesenchymal stem cells capable of hematopoietic progenitor cell expansion. J Exp Med. 2013;210:1351-67.

28. Nakashima K, Zhou X, Kunkel G, et al. The novel zinc fingercontaining transcription factor osterix is required for osteoblast differentiation and bone formation. Cell. 2002;108:17-29.

29. Zhang J, Niu C, Ye L, et al. Identification of the haematopoietic stem cell niche and control of the niche size. Nature. 2003;425:836-41.

30. Calvi LM, Adams GB, Weibrecht KW, et al. Osteoblastic cells regulate the haematopoietic stem cell niche. Nature. 2003;425:841-6.

31. Visnjic D, Kalajzic Z, Rowe DW, et al. Hematopoiesis is severely altered in mice with an induced osteoblast deficiency. Blood. 2004;103:3258-64.

32. Mizoguchi T, Pinho S, Ahmed J et al. Osterix marks distinct waves of primitive and definitive stromal progenitors during bone marrow development. Dev Cell 2014 (in press).

33. Owen M, Friedenstein AJ. Stromal stem cells: marrow-derived osteogenic precursors. Ciba Found Symp. 1988;136:42-60.

34. Hanoun M, Frenette PS. This niche is a maze; an amazing niche. Cell Stem Cell. 2013;12:391-2.

35. Ito $\mathrm{K}$, Bernardi $\mathrm{R}$, Morotti $\mathrm{A}$, et al. PML targeting eradicates quiescent leukaemia-initiating cells. Nature. 2008;453:1072-8.

36. Matsuoka Y, Sasaki Y, Nakatsuka R, et al. Low level of c-kit expression marks deeply quiescent murine hematopoietic stem cells. Stem Cells. 2011;29:1783-91.

37. Venezia TA, Merchant AA, Ramos CA, et al. Molecular signatures of proliferation and quiescence in hematopoietic stem cells. PLoS Biol. 2004;2:e301.

38. Shin JY, Hu W, Naramura M, Park CY. High c-kit expression identifies hematopoietic stem cells with impaired self-renewal and megakaryocytic bias. J Exp Med. 2014;211:217-31.

39. Mazo IB, Gutierrez-Ramos JC, Frenette PS, et al. Hematopoietic progenitor cell rolling in bone marrow microvessels: parallel contributions by endothelial selectins and vascular cell adhesion molecule 1. J Exp Med. 1998;188:465-74.

40. Frenette PS, Mayadas TN, Rayburn H, et al. Susceptibility to infection and altered hematopoiesis in mice deficient in both Pand E-selectins. Cell. 1996;84:563-74. 
41. Laird DJ, von Andrian UH, Wagers AJ. Stem cell trafficking in tissue development, growth, and disease. Cell. 2008;132:612-30.

42. Scheiermann C, Kunisaki Y, Lucas D, et al. Adrenergic nerves govern circadian leukocyte recruitment to tissues. Immunity. 2012;37:290-301.
43. Winkler IG, Barbier V, Nowlan B, et al. Vascular niche E-selectin regulates hematopoietic stem cell dormancy, self renewal and chemoresistance. Nat Med. 2012;18:1651-7. 